\title{
A utilização de VPN para integrar WoT em uma assistente virtual ${ }^{*}$
}

\author{
Julianne Amanda de Sá Laurindo ${ }^{1}$, \\ Antonio Deusany de Carvalho Junior ${ }^{2}$, Alfredo Goldman ${ }^{2}$ \\ ${ }^{1}$ Escola de Artes, Ciências e Humanidades - EACH - USP \\ Rua Arlindo Béttio, 1000 - São Paulo - SP - Brazil \\ ${ }^{2}$ Instituto de Matemática e Estatística - IME - USP \\ Rua do Matão, 1010 - São Paulo - SP - Brazil \\ julianne@usp.br, $\{d j, g \circ l d\} @ i m e . u s p . b r$
}

\begin{abstract}
The Mozilla Webthings Gateway presents some limitation: it is only possible to control devices that belong to the same Internet network, in other words, using a local network. In this work we tackled this situation with a virtual local network, a VPN, and incorporated a virtual assistant A.D.A. to the environment. The VPN chosen and evaluated thorughout this research was OpenVPN. Thus, it was validated a possible integration with Web of Things into A.D.A. on distributed environments with promising results.
\end{abstract}

Resumo. O Gateway Mozilla Webthings possui uma limitação: só é possível controlar dispositivos que pertencem à mesma rede de Internet, ou seja, utilizando uma rede local. Neste trabalho resolvemos esta limitação através de uma rede local virtual, uma VPN, e incorporamos uma assistente pessoal, A.D.A., ao ambiente. A VPN escolhida e avaliada durante esta pesquisa foi baseada no OpenVPN. Com isso, validamos uma possível solução para a integração de Web of Things na A.D.A. em ambientes distribuídos com resultados promissores.

\section{Introdução}

A A.D.A., assistente distribuída avançada, é um projeto iniciado em 2019 na Universidade de São Paulo para a criação de uma assistente pessoal distribuída inteligente, a qual tem como objetivo interagir com o usuário, em português, conectando-o com dispositivos através da Internet das Coisas (Internet of Things - IoT) [Freire et al. 2020]. Adicionalmente, pretende ser uma assistente baseada em microsserviços e de código aberto $^{1}$. A primeira fase do projeto resultou em diversos artigos sobre funcionalidades, frameworks, API's e tecnologias para serem utilizadas na elaboração da assistente virtual [Marques et al. 2020, da Silva et al. 2020, Evangelista et al. 2020, Neto et al. 2020, de Andrade et al. 2020].

Neste trabalho, o foco foi a validação do uso de VPN na ADA. A VPN serve neste caso para facilitar a interconexão entre dispositivos distribuídos em ambientes diferentes. Tal validação visa verificar a possibilidade de utilização do gateway Web of Things para facilitar a comunicação entre os dispositivos dentro de uma VPN. Por fim, são descritas as tecnologias utilizadas e a arquitetura final implementada.

*Este projeto foi financiado pelo Conselho Nacional de Desenvolvimento Científico e Tecnológico (CNPq) e pelo grupo de extensão USPCodeLab uclab. xyz/site

${ }^{1}$ Repositório da A.D.A.: https://gitlab.com/advanceddistributedassistant 


\section{Web of Things}

O WebofThings foi introduzido na ADA por apresentar vantagens com relação à sua padronização com a W3C, uma comunidade focada em criar padrões para internet. Uma de suas vantagens é o uso de protocolos mais comuns na internet para conectar os dispositivos às redes, o que não é comum em outras arquiteturas. Segundo a W3C, as vantagens do WebofThings vão além, como uma aplicação mais segura, simples, flexível e reutilizável [Gupta et al. 2011]. Essa ferramenta tem sua interação baseada em propriedades, eventos e ações. As propriedades são os estados dos objetos, ligado ou desligado, valores de sensores, etc. Já as ações são os comandos para alteração dos estados dos objetos, como por exemplo, ligar um motor ou desligá-lo. E por fim, os eventos são a descrição da alteração do estado, por exemplo, porta aberta.

O WebThings Gateway ${ }^{2}$, pertencente a Mozilla, foi acrescentado ao projeto por ser open source, seguir os padrões do W3C e por ter uma boa documentação. Com seu uso, é possível a criação de quantas contas forem necessárias e nestas contas, existe a capacidade de adicionar serviços, tais quais os protocolos a serem utilizados ou até mesmo API's. O Gateway da Mozilla permite a criação de ambientes para a separação dos dispositivos da casa. Ademais, é capaz de criar uma planta baixa da sua casa para a melhor visualização da distribuição de seus dispositivos. Contudo, foi constatado que esse framework não é distribuído, o que é algo necessário para a elaboração da A.D.A.

Para manter viável a utilização o WebThings Gateway no projeto foi constatada a possibilidade da utilização de uma Virtual Private Network (VPN). Esta é amplamente utilizada na comunidade de jogos, o que estimulou seu uso também neste trabalho. $\mathrm{O}$ incentivo para a utilização dessa tecnologia é seu baixo custo, tendo um bom desempenho de criar rotas na rede pública para acessar as máquinas. [Harmening 2017]. A VPN criptografa os dados enviados como forma de segurança, isto é os desordena através de um algoritmo ou cifra e transforma em algo incompreensível, se visto sem a chave, que é a senha para ordenar e desordenar os dados.

A escolha pela tecnologia de VPN em detrimento de outras, como o cloud computing e Zero Trust Network Access (ZTNA), se deu pela sua praticidade para interconexão remota, e sua avaliação para este setup foi bem sucedida. No mundo dos jogos digitais, por exemplo, VPNs são muito utilizadas para a simulação de redes locais, resultando em uma grande quantidade de conteúdo acessível sobre como usá-las. Sendo assim, da mesma maneira que ocorre nos ambientes de jogos em rede, a latência em uma VPN existe, mas pode não ser tão perceptível dependendo das ações a serem realizadas.

\section{Metodologia}

Conforme citado anteriormente, para dirimir o problema do gateway da Mozilla optou-se pela sua utilização em uma VPN. Neste trabalho foram realizados testes para controlar um LED a partir de um computador em outro ambiente a fim de validar a comunicação por meio da VPN com o WebOfThings. O teste inicial funcionou duas vezes, contudo não foi possível repetir o teste em outras máquinas utilizando a primeira VPN escolhida, o LogMeIn Hamachi, pois foram encontrados diversos erros, e por isso, foi decidido a utilização de uma outra VPN, OpenVPN, a qual é open source, que faz jus ao proposito da ADA.

\footnotetext{
${ }^{2}$ WebThings Gateway: https://webthings .io/
} 
A VPN foi instalada, no raspberry que utilizava sistema operacional Raspberry Pi OS, através do terminal pelo comando "curl -L https://install.pivpn.io — bash". Durante a instalação, foram configuradas diversas opções, como o algoritmo de criptografia e o tipo de criptografia. Neste caso, a escolhida foi a de 256 bits, que é a mais recomendada. São dados informações a serem utilizadas posteriormente para a configuração como o IP e a porta da rede. Além disso, foi definido o protocolo de rede, no caso, o TCP. Este processo gerou uma chave, a qual foi salva em um dispositivo de armazenamento. Depois, o computador foi reniciado para completar o processo e no terminal foi usado o comando "pivpn ovpn -a". A seguir, foram definidos o nome e a senha do cliente. Posteriormente, foram configurados também um DNS dinâmico e o port forwarding, os quais permitem a atribuição de um domínio fixo a um endereço IP da rede dinâmico e o acesso a rede mesmo estando fora dela, respectivamente.

Após isto, foi instalado o OpenVPN em outra máquina fora da rede inicial e inserido o Pendrive que possuía a chave, tendo apenas que digitar a senha criada em um passo anterior para finalizar a interconexão. Uma representação da arquitetura final pode ser vista na Figura 1. Visando, ainda, a simplicidade do sistema, seu setup foi pensado para que qualquer computador pessoal possa ser utilizado para replicar sua arquitetura.

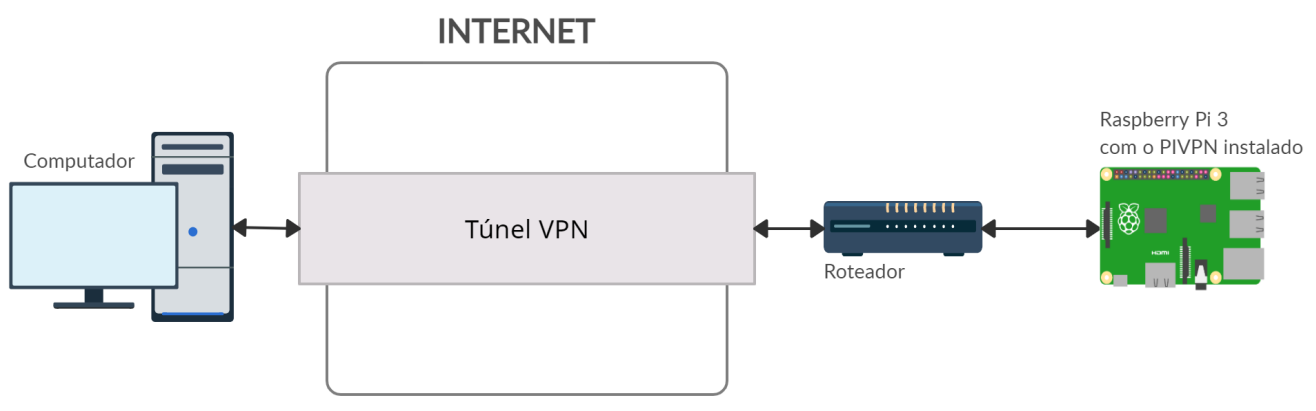

Figura 1. Arquitetura de Testes da VPN para a A.D.A.

\section{Resultados e discussão}

Os dispositivos foram conectados com sucesso por meio da VPN, após as dificuldades enfrentadas. Dentre tais dificuldades, podemos citar o desafio de entender como se instala e utiliza o OpenVPN, quais configurações eram melhores, criptografias disponíveis, além de outras definiçõos técnicas que as demais VPNs, como o Hamachi, fazem de forma transparente para o usuário. Entretanto, após a configuração, é simples a instalação e o acesso em qualquer outro dispositivo na rede. O ambiente foi testado com os sistemas operacionais Windows e Linux sem muito problema.

Houve sucesso ao acessar a rede externa, e foi possível testar o tempo de envio de pacotes entre dispositivos conectados. Em média, o ping foi de $38 \mathrm{~ms}$, sendo que a rede utilizada se encontrava em Curitiba e o dispositivo conectado estava localizado na cidade de São Paulo. No fim, não foi possível testar funcionalidades diversas do Mozilla WebThings Gateway. Todavia, se tornou possível acessar e controlar os dispositivos a partir de uma rede local distinta. Esse resultado é de grande relevância para o desenvolvimento da ADA, visto que ela objetiva ser um sistema distribuído e de simples administração. 


\section{Conclusão}

Foi possível concluir que uma vantagem do uso da VPN para a A.D.A. é a oportunidade de interagir com esta em diferentes locais, como em casa e no trabalho, tendo só uma conta para o controle de tudo em uma mesma rede virtual. Além disso, outra vantagem é que a ferramenta permite o controle sem estar no mesmo ambiente para trocar o estado do objeto, tendo como exemplo, ligar e desligar uma lâmpada enquanto viaja.

Uma das possibilidades trazidas pela integração distribuída é a facilidade de compartilhamento de dados entre as máquinas, no caso o acesso aos objetos e suas mudanças de estado. Com isso, temos também que a interação entre usuário e dispositivo torna-se mais simples, pois o controle de toda a smart home é feito por apenas uma plataforma.

\section{Referências}

da Silva, A. C. S., de Oliveira Rosa, T., Scattone, F. F., and Goldman, A. (2020). Um estudo exploratório sobre infraestrutura de operação para uma assistente virtual inteligente. In Anais da XI Escola Regional de Alto Desempenho de São Paulo. SBC.

de Andrade, A., Moura, S., and Goldman, A. (2020). Processamento e transcrição de voz em língua portuguesa voltado para assistente inteligente. In Anais da I Escola Regional de Aprendizado de Máquina e Inteligência Artificial de São Paulo, pages 1-4. SBC.

Evangelista, L. A., do Nascimento, P., Elmadjian, C. E., and Lejbman, A. V. (2020). Uma revisão de arquiteturas ponta a ponta para sintetização de voz. In Anais da I Escola Regional de Aprendizado de Máquina e Inteligência Artificial de São Paulo, pages 17-21. SBC.

Freire, F., Rosa, T., Feulo, G., Elmadjian, C., Cordeiro, R., Moura, S., Andrade, A., de Omena, L. A., Vicente, A., Marques, F., Sheffer, A., Hideki, O., Nascimento, P., Cordeiro, D., and Goldman, A. (2020). Toward Development of A.D.A. - Advanced Distributed Assistant. In Anais do XXI Simpósio em Sistemas Computacionais de Alto Desempenho, pages 203-214, Porto Alegre, RS, Brasil. SBC.

Gupta, V., Goldman, R., and Udupi, P. (2011). A network architecture for the web of things. In Proceedings of the Second International Workshop on Web of Things, WoT '11, New York, NY, USA. Association for Computing Machinery.

Harmening, J. T. (2017). Virtual private networks. In Computer and Information Security Handbook, pages 843-856. Elsevier.

Marques, F., Hideki, O., and Goldman, A. (2020). Escalonamento de operações em dispositivos iot para assistentes virtuais. In Anais da XI Escola Regional de Alto Desempenho de São Paulo. SBC.

Neto, A. V., do Espírito Santo, G., and Goldman, A. (2020). Compreensão de linguagem natural: Uma solução em português brasileiro. In Anais da I Escola Regional de Aprendizado de Máquina e Inteligência Artificial de São Paulo, pages 5-8. SBC. 